Research Paper: Pathology

\title{
Zerumbone ameliorates high-fat diet-induced adiposity by restoring AMPK-regulated lipogenesis and microRNA-146b/ SIRT1-mediated adipogenesis
}

\author{
Jiyun Ahn ${ }^{1,2}$, Hyunjung Lee ${ }^{1}$, Chang Hwa Jung ${ }^{1,2}$, Won Hee Choi ${ }^{1}$ and Tae Youl Ha ${ }^{1,2}$ \\ ${ }^{1}$ Research Group of Metabolic Mechanism, Korea Food Research Institute, Seongnam, Korea \\ 2 Division of Food Biotechnology, University of Science \& Technology, Daejeon, Korea \\ Correspondence to: Tae Youl Ha, email: tyhap@kfri.re.kr \\ Keywords: obesity; zerumbone; SIRT1; microRNA-146b; adipogenesis; Pathology Section \\ Received: October 24, $2016 \quad$ Accepted: March 14, $2017 \quad$ Published: April 08, 2017 \\ Copyright: Ahn et al. This is an open-access article distributed under the terms of the Creative Commons Attribution License (CC-BY), which \\ permits unrestricted use, distribution, and reproduction in any medium, provided the original author and source are credited.
}

\section{ABSTRACT}

Obesity is characterized by increased fat mass, as adipose tissue serves as a storage site for excess energy from food consumption. In obesity, altered lipid metabolism of adipose tissue, characterized by fatty acid uptake, de novo lipogenesis, and lipolysis, are induced. In this study, we examined the effect of zerumbone, a major sesquiterpene from wild ginger, on high-fat diet (HF)-induced obesity and dysregulated lipid metabolism in the white adipose tissues (WAT) of C57BL/6N mice. Dietary supplementation with zerumbone ameliorated HF-induced obesity and improved impaired lipid metabolism in WAT. Zerumbone additionally induced AMPK activation and phosphorylation of acetyl-CoA carboxylase, and effectively decreased adipogenic differentiation, in a concentration-dependent manner in the 3T3-L1 cells. Dysregulated microRNAs in obese WAT and adipocytes were examined, and zerumbone treatment was found to effectively reverse the robust upregulation of microRNA-146b. An increase in the levels of SIRT1, the direct target of microRNA146b, was observed in zerumbone-treated differentiated adipocytes. This increase was additionally observed in WAT of zerumbone-supplemented mice. The antiadipogenic effect of zerumbone was found to be abolished in SIRT1-silenced 3T3-L1 cells. The increase in SIRT1 levels induced by zerumbone led to deacetylation of FOXO1 and PGC1a in WAT and differentiated 3T3-L1 cells. These findings indicate that zerumbone ameliorated diet-induced obesity and inhibited adipogenesis, and that the underlying mechanisms involved AMPK and the microRNA-146b/SIRT1 pathway. Zerumbone may represent a potential therapeutic candidate for the prevention and treatment of metabolic diseases, particularly obesity.

\section{INTRODUCTION}

Obesity is an energy imbalance disorder in which nutrient intake chronically exceeds energy expenditure, resulting in the increased fat mass [1]. Obesity is the primary risk factor for metabolic syndrome, which is characterized by hypertension, type 2 diabetes, and cardiovascular disease [2]. The worldwide prevalence of obesity has more than doubled since 1980 . According to WHO statistics, $39 \%$ of adults over 18 years old were overweight, and $13 \%$ were obese in 2014 . Unless the current trend is reversed, the continuing increase in obesity will have detrimental effects on global health and economy. Despite advances in weight management strategies in recent years, the treatment of obesity remains a significant challenge. The potential of natural phytochemicals as substitutes for weight management [3] and their ability to increase fatty acid $\beta$-oxidation, fat absorption, and suppress appetite [4] have received much interest.

Zerumbone, a cyclic sesquiterpene present in rhizomes of the wild ginger Zingiber zerumbet Smith, has been identified as a tumor suppressor $[5,6]$ in various cancer cells. Zerumbone induces cell cycle 
arrest [7], inhibits TGF- $\beta 1$ signaling [8, 9], and inhibits tumor angiogenesis by blocking $\mathrm{NF}-\kappa \mathrm{B}$ signaling [10]. In addition, zerumbone exhibits a wide range of pharmacological activities such as anti-cataractogenesis [11], anti-inflammatory effects [12], and anti-viral effects [13]. Zerumbone has also been shown to attenuate nonalcoholic fatty liver [14], mitigate hypertriglyceridemia [15], and ameliorate diabetic nephropathy [16]. However, the effect of zerumbone on obesity has not been reported to date.

MicroRNAs (miRNAs) are highly conserved, small non-coding RNAs that regulate gene expression at the post-transcriptional level by binding to complementary sites on their target transcripts and either inducing mRNA cleavage or repressing protein translation [17]. The dysregulation of miRNA is important in cancer initiation and progression [18]. The miRNAs have emerged as important post-transcriptional regulators of adipocyte metabolism, energy homeostasis, lipid metabolism, pancreatic $\beta$-cell development, and weight gain resulting from a high-fat diet [19-22]. Among them, miR-27a and miR-27b have been reported as negative regulators of adipocyte differentiation through peroxisome proliferatoractivated receptor gamma (PPAR $\gamma$ ) inhibition [23]. Natural compounds such as persimmon tannin have been shown to inhibit the differentiation of 3T3-L1 cells through upregulation of miR-27a and miR-27b [24]. We previously reported that miR-146b functions as a positive regulator of adipocyte differentiation through downregulation of sirtuin 1 (SIRT1) [25]. However, chemicals from natural sources that could downregulate miR-146b were not investigated.

In this study, we examined the effect of zerumbone on high-fat diet-induced obesity and measured the expression levels of lipid metabolism-regulated genes in adipose tissue in mice and differentiated 3T3-L1 cells. We also measured the effect of zerumbone on 5' AMPactivated protein kinase (AMPK) phosphorylation. To investigate the role of miRNA in the anti-obesity effects of zerumbone, we analyzed miRNA expression profiles in adipose tissue and performed a functional study to validate miRNA-target mRNA interactions.

\section{RESULTS}

\section{Zerumbone decreased adiposity in C57BL/6N mice with diet-induced obesity}

To determine the effect of zerumbone on obesity, C57BL/6N mice were fed a high-fat diet (HF) containing $0.01 \%(\mathrm{HF}+\mathrm{LZ})$ or $0.025 \%(\mathrm{HF}+\mathrm{HZ})$ zerumbone for 8 weeks (Figure 1A). Mice fed a normal control diet (NC) gained $12.59 \pm 0.46 \mathrm{~g}$, whereas the HF-fed mice showed a significantly higher body weight gain of $20.81 \pm 1.01 \mathrm{~g}$ (Figure 1B). However, $\mathrm{HF}+\mathrm{LZ}$ and $\mathrm{HF}+\mathrm{HZ}$ resulted in lower weight gain (18.2\% and $25.1 \%$, respectively) than that in the HF group $(P<0.05)$. Food intake did not differ between the groups (Figure 1C). The weight of epididymal white adipose tissue (WAT) was significantly reduced in the zerumbone-supplemented mice compared with the HF mice $(P<0.05)$ (Figure 1D). Histological examination of WAT showed that $\mathrm{HF}+\mathrm{HZ}$ significantly decreased adipocyte size (Figure 1E and F). Blood profile measurements showed that HF-induced hypertriglycemia, hyperleptinemia, hyperinsulinemia, hyperglycemia, and increase in FFA and homeostasis model assessmentestimated insulin resistance (HOMA-IR) were all significantly improved following zerumbone treatment (Table 1).

The alanine transaminase (ALT) and aspartate transaminase (AST) are standard biomarkers for druginduced liver disease [26]. To evaluate zerumbone-induced hepatotoxicity, we measured the AST/ALT activity in the liver tissues and found that zerumbone did not induce liver toxicity (Figure $1 \mathrm{G}$ and $1 \mathrm{H}$ ).

\section{Zerumbone ameliorated dysregulated lipid metabolism in WAT}

We analyzed the effect of zerumbone supplementation on lipid metabolism in WAT. HF feeding altered the lipid metabolism of WAT with upregulation of adipogenic and lipogenic genes, as well as downregulation of fatty acid oxidation-regulating genes (Figure 2A). We observed that zerumbone treatment effectively reversed the changes in the expression of genes involved in lipid metabolism in WAT.

Activation of AMPK in adipose tissue limits fatty acid efflux from adipocytes and favors fatty acid oxidation [27]. In addition, AMPK decreases de novo fatty acid synthesis through phosphorylation-mediated inhibition of acetyl-CoA carboxylase (ACC) [28]. Given the important role of AMPK in the regulation of adipose tissue metabolism, we evaluated the effect of zerumbone-induced AMPK activation on adipose metabolism. As shown in Figure 2B, zerumbone increased the phosphorylation of AMPK in WAT; however, the total AMPK content of the experimental groups was similar. The increase in AMPK phosphorylation corresponded with increased ACC (Figure 2C and 2D).

\section{Zerumbone inhibited adipogenesis in 3T3-L1 cells}

As zerumbone has been reported to induce apoptotic cell death in several cancer cell lines [29, 30], we tested the cytotoxic effects of zerumbone, and found that it did not exert cytotoxicity up to a concentration of 50 $\mu \mathrm{M}$ (Figure 3A). Zerumbone inhibited adipogenesis in a concentration-dependent manner (Figure 3B and 3C). Differentiation-induced lipid accumulation was 
accompanied by upregulation of the master adipogenic transcription factors CCAAT enhancer binding protein alpha $(\mathrm{C} / \mathrm{EBP} \alpha)$ and PPAR $\gamma$. Zerumbone inhibited the induction of these transcription factors as well as that of fatty acid synthase (FASN, a downstream target of PPAR $\gamma$ ) and adipocyte protein 2 (Ap2, a carrier protein expressed in adipocytes) in both mRNA and protein levels (Figure $3 \mathrm{D}$ and $3 \mathrm{E})$. These results suggest that zerumbone inhibits adipogenic differentiation via downregulation of $\mathrm{C} / \mathrm{EBP} \alpha$ and PPAR $\gamma$.

\section{Zerumbone reversed the dysregulated miRNA expression in WAT and 3T3-L1 cells}

MiRNAs play regulatory roles in obesity by regulating adipocyte differentiation, insulin activity, and fat metabolism [31]. The miRNA expression profiling in obese and lean WAT from mice has revealed that miRNAs are dysregulated in obese adipose tissue [32-34]. Based on this evidence, we selected 21 dysregulated miRNAs and compared their expression in WAT from NC, HF, and $\mathrm{HF}+\mathrm{Z}$ groups (Table 2). The 10 most upregulated miRNAs were miR-146b, miR-297b, miR-34a, miR-469,
A

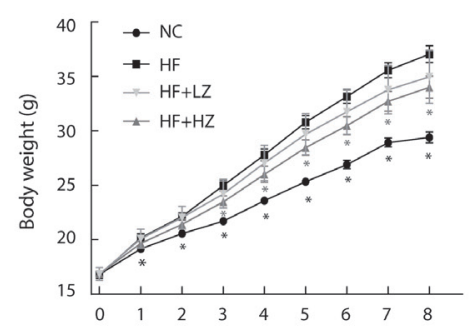

D

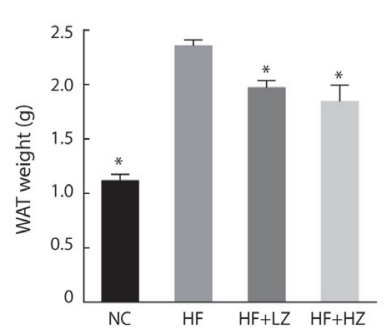

$\mathrm{F}$

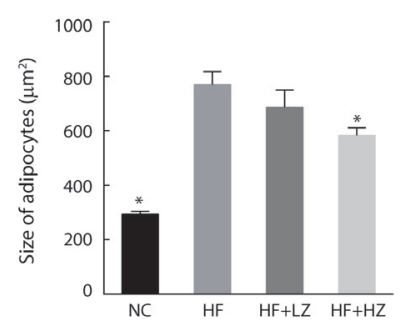

B

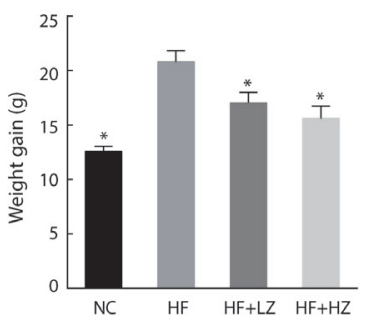

E
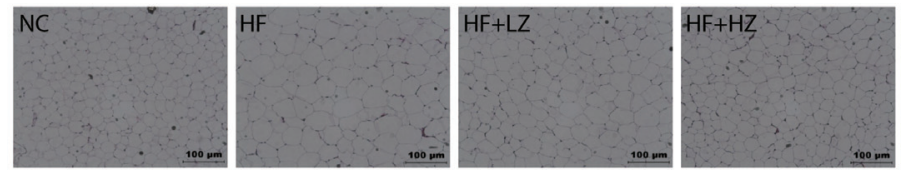

G

$\mathrm{H}$

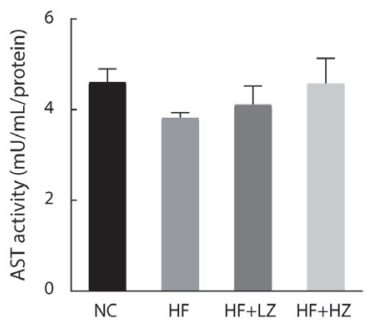

C

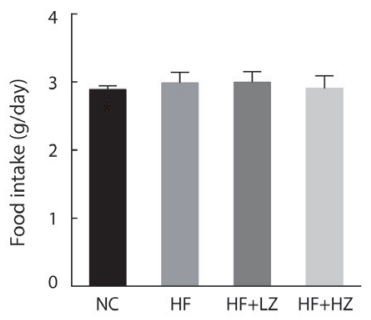

$$
\text { (1) }
$$


Table 1: Effect of zerumbone on serum glucose and lipid profiles

\begin{tabular}{|l|c|c|c|c|}
\hline & NC & HF & HF + LZ & HF + HZ \\
\hline Triglyceride $(\mathrm{mg} / \mathrm{dL})$ & $48.13 \pm 4.07^{*}$ & $53.59 \pm 1.74$ & $40.32 \pm 1.38^{*}$ & $37.21 \pm 2.11^{*}$ \\
\hline TC $(\mathrm{mg} / \mathrm{dL})$ & $81.24 \pm 7.15^{*}$ & $128.97 \pm 138.08$ & $126.66 \pm 7.15$ & $133.99 \pm 11.24$ \\
\hline HDL-C $(\mathrm{mg} / \mathrm{dL})$ & $43.56 \pm 6.07^{*}$ & $50.30 \pm 59.22$ & $60.11 \pm 12.79$ & $68.89 \pm 6.40^{*}$ \\
\hline HDL-C/TC & $0.53 \pm 0.11^{*}$ & $0.38 \pm 0.44$ & $0.46 \pm 0.07$ & $0.49 \pm 0.04$ \\
\hline FFA $(\mu \mathrm{Eq} / \mathrm{L})$ & $512.40 \pm 67.02^{*}$ & $784.30 \pm 16.27$ & $601.9 \pm 28.35$ & $453.90 \pm 7.03^{*}$ \\
\hline Leptin $(\mathrm{ng} / \mathrm{mL})$ & $23.89 \pm 7.95^{*}$ & $90.61 \pm 45.05$ & $61.52 \pm 13.20^{*}$ & $55.47 \pm 6.36^{*}$ \\
\hline Insulin $(\mathrm{ng} / \mathrm{mL})$ & $0.30 \pm 0.07^{*}$ & $0.60 \pm 0.39$ & $0.44 \pm 0.11^{*}$ & $0.31 \pm 0.05^{*}$ \\
\hline Glucose $(\mathrm{mg} / \mathrm{dL})$ & $264.76 \pm 48.29^{*}$ & $501.60 \pm 41.18$ & $387.70 \pm 23.67^{*}$ & $329.20 \pm 30.45^{*}$ \\
\hline HOMA-IR & $4.89 \pm 0.42^{*}$ & $10.39 \pm 1.56$ & $6.85 \pm 0.67^{*}$ & $6.47 \pm 0.39^{*}$ \\
\hline
\end{tabular}

Each group consisted of 10 mice. Results are the means \pm SEM. $* P<0.05$ versus HF group.

$\mathrm{NC}$, normal control diet-fed mice; HF, high-fat diet fed mice; $\mathrm{HF}+\mathrm{LZ}$, high-fat diet containing $0.01 \%$ zerumbone-fed mice; $\mathrm{HF}+\mathrm{HZ}$, high-fat diet containing $0.025 \%$ zerumbone-fed mice

TC, total cholesterol; HDL-C, high density lipoprotein-cholesterol; FFA, free fatty acid; HOMA-IR, homeostasis model assessment-estimated insulin resistance.

miR-139-3p, miR-21, miR-466E-5p, miR22*, miR-324, and miR-143. We additionally measured the expression of these 10 miRNAs in the differentiated 3T3-L1 cells. miR$146 \mathrm{~b}$ exhibited the highest upregulation, with a 3.39-fold increase in the WAT of HF mice and an 8.12-fold increase in differentiated 3T3-L1 cells. Interestingly, zerumbone treatment effectively reversed the dysregulation of miR$146 \mathrm{~b}$ expression in WAT from HF and in differentiated 3T3-L1 cells. These results suggest that zerumbone is a negative regulator of miR-146b in WAT from obese mice and in differentiated adipocytes.

\section{Anti-adipogenic effect of zerumbone is dependent on SIRT1, a direct target of miR-146b}

To evaluate the effect of zerumbone on miR-146b expression, we treated fully differentiated 3T3-L1 cells with zerumbone or miR-146b inhibitor. Zerumbone inhibited miR-146b expression similar to that observed with miR-146b inhibitor treatment (Figure 4A). Conversely, when 3T3-L1 cells were exposed to a miR$146 \mathrm{~b}$ mimic and zerumbone simultaneously, zerumbone inhibited the upregulation of miR-146b caused by the miR-146b mimic (Figure 4B). miRNA induces repression

A

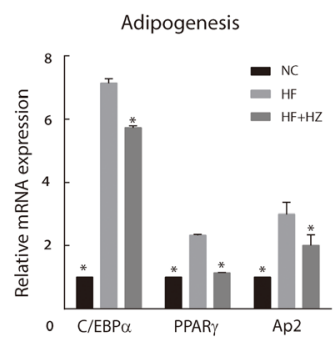

B

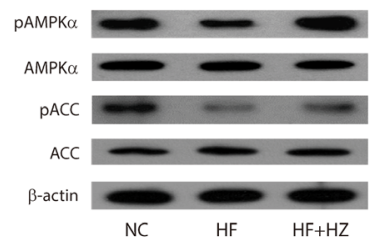

Fatty acid oxidation

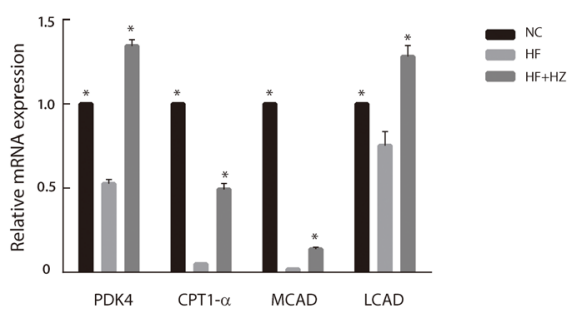

C

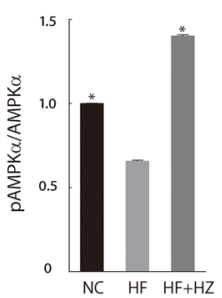

De novo lipogenesis

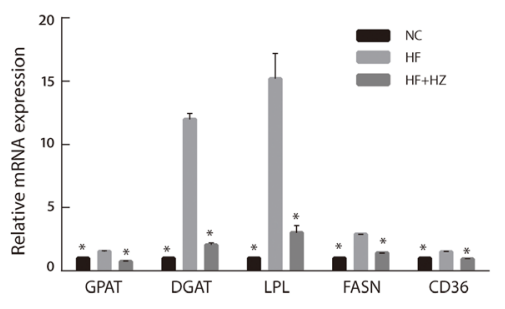

D

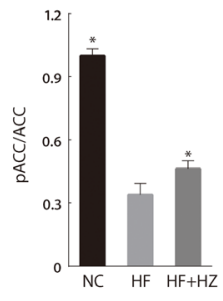

Figure 2: Zerumbone inhibited adipogenesis and modulated lipid metabolism in WAT. A. Effect of zerumbone on mRNA levels of lipid metabolism-related genes in WAT from mice fed NC, HF, and HF + HZ. B. Effect of zerumbone on AMPK signaling in WAT was determined by western blot. C. Ratio of phosphorylated AMPK to total AMPK in WAT. D. Ratio of phosphorylated ACC to total ACC in WAT. ${ }^{*} P<0.05$ versus HF. 
Table 2: Effect of zerumbone on microRNA expressions in differentiated 3T3-L1 cells and C57BL/6N mice

\begin{tabular}{|l|c|c|c|c|}
\hline & $\mathbf{H F}$ & $\mathbf{H F}+\mathbf{Z}$ & $\mathbf{D}$ & $\mathbf{D}+\mathbf{Z}$ \\
\hline mmu-miR-146b & $\mathbf{3 . 3 9} \pm \mathbf{0 . 0 3}$ & $\mathbf{1 . 8 4} \pm \mathbf{0 . 0 2}$ & $\mathbf{8 . 1 2} \pm \mathbf{0 . 8 5}$ & $\mathbf{3 . 4 3} \pm \mathbf{0 . 0 6 ^ { \# }}$ \\
\hline mmu-miR-297b & $3.18 \pm 0.01$ & $1.23 \pm 0.01^{*}$ & $0.58 \pm 0.01$ & $1.28 \pm 0.03^{\#}$ \\
\hline mmu-miR-34a & $3.14 \pm 0.02$ & $2.58 \pm 0.06^{*}$ & $6.93 \pm 0.34$ & $1.90 \pm 0.04^{\#}$ \\
\hline mmu-miR-469 & $2.87 \pm 0.03$ & $1.46 \pm 0.15^{*}$ & $0.46 \pm 0.26$ & $3.35 \pm 0.11^{\#}$ \\
\hline mmu-miR-139-3p & $2.63 \pm 0.03$ & $1.20 \pm 0.05^{*}$ & $0.03 \pm 0.0$ & $0.54 \pm 0.02^{\#}$ \\
\hline mmu-miR-21 & $2.50 \pm 0.21$ & $2.24 \pm 0.2$ & $6.17 \pm 0.25$ & $1.06 \pm 0.06^{\#}$ \\
\hline mmu-miR-466E & $2.40 \pm 0.01$ & $0.98 \pm 0.01^{*}$ & $0.57 \pm 0.01$ & $1.07 \pm 0.04^{\#}$ \\
\hline mmu-miR-22* & $2.18 \pm 0.01$ & $1.68 \pm 0.20^{*}$ & $0.35 \pm 0.02$ & $0.53 \pm 0.00^{\#}$ \\
\hline mmu-miR-324 & $2.08 \pm 0.01$ & $1.53 \pm 0.01^{*}$ & $0.32 \pm 0.01$ & $0.51 \pm 0.03^{\#}$ \\
\hline mmu-miR-143 & $1.93 \pm 0.03$ & $1.53 \pm 0.07^{*}$ & $1.27 \pm 0.02$ & $0.95 \pm 0.01^{\#}$ \\
\hline mmu-miR-720 & $1.59 \pm 0.03$ & $1.29 \pm 0.05^{*}$ & & \\
\hline mmu-miR-15b & $1.46 \pm 0.04$ & $0.56 \pm 0.03^{*}$ & & \\
\hline mmu-miR-302a & $1.42 \pm 0.01$ & $0.92 \pm 0.03^{*}$ & & \\
\hline mmu-miR-466h & $1.34 \pm 0.04$ & $1.48 \pm 0.06^{*}$ & & \\
\hline mmu-miR-197 & $1.31 \pm 0.01$ & $1.09 \pm 0.05^{*}$ & & \\
\hline mmu-miR-423 & $1.27 \pm 0.05$ & $0.96 \pm 0.05^{*}$ & & \\
\hline mmu-miR-715 & $1.25 \pm 0.01$ & $1.31 \pm 0.16^{*}$ & & \\
\hline mmu-miR-467 & $1.24 \pm 0.00$ & $1.63 \pm 0.1^{*}$ & & \\
\hline mmu-miR-709 & $0.97 \pm 0.01$ & $0.73 \pm 0.03^{*}$ & & \\
\hline mmu-miR-122 & $0.69 \pm 0.04$ & $0.05 \pm 0.06^{*}$ & & \\
\hline mmu-miR-802 & $0.37 \pm 0.01$ & $2.76 \pm 0.10^{*}$ & & \\
\hline
\end{tabular}

Results are the means \pm SD. For epididymal adipose tissue, expressions of miRNAs are shown as fold-change relative to the level of NC group. ${ }^{*} P<0.05$ versus HF group. For 3T3-cells, expressions of miRNAs are shown as fold-change relative to the level of non-differentiated preadipocytes. ${ }^{\#} P<0.05$ versus $\mathrm{D}$ group.

$\mathrm{HF}$, high-fat diet-fed mice; $\mathrm{HF}+\mathrm{Z}$, high-fat diet containing $0.025 \%$ zerumbone-fed mice; $\mathrm{D}$, differentiated adipocytes; $\mathrm{D}+\mathrm{Z}$, differentiated adipocytes treated with10 $\mu \mathrm{M}$ zerumbone.

A

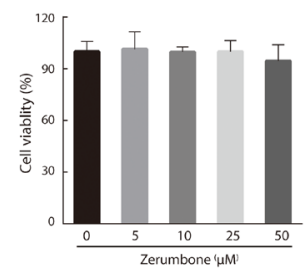

D

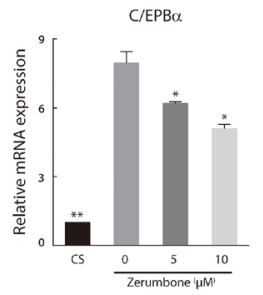

B

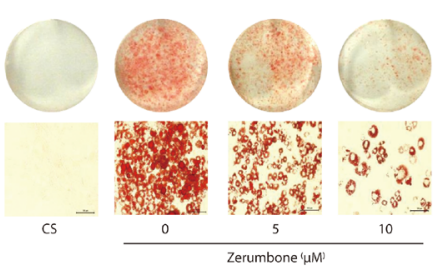

PPARY

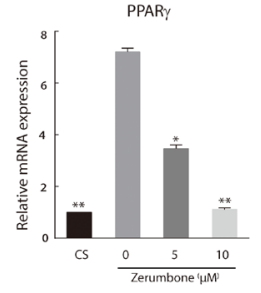

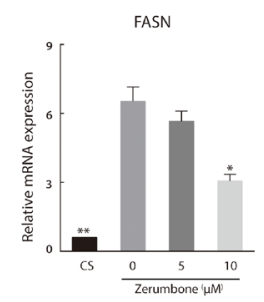

C

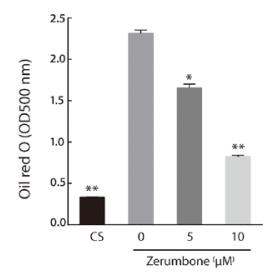

$\mathrm{E}$

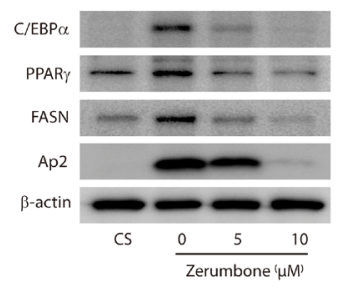

Figure 3: Anti-adipogenic effect of zerumbone in 3T3-L1 cells. A. Effect of zerumbone on cell viability after $48 \mathrm{~h}$ exposure. B. Oil Red O staining of 3T3-L1 cells that were differentiated for 10 days. Cells were treated with 5 or $10 \mu \mathrm{M}$ zerumbone for $48 \mathrm{~h}$ at day 0 . Non-differentiated preadipocytes (CS) were incubated in DMEM with $10 \%$ calf serum. Representative photomicrographs $(\times 200)$ are shown for each treatment group. C. Intracellular lipid accumulation was quantified by measuring optical absorbance at $500 \mathrm{~nm}$. D. Real-time PCR analysis of adipogenic transcription factors and their targets in 3T3-L1 cells. The level of each mRNA is expressed relative to the average level of CS. ${ }^{*} P<0.05,{ }^{*} P<0.01$ versus differentiated adipocytes. E. Western blotting was performed for various adipocyte differentiation markers. 
of protein translocation or degradation of mRNA through imperfect complementary binding between the miRNA and target mRNA [35]. Therefore, we investigated the effect of zerumbone on the binding activity of miR-146b. Previously, we reported that miR-146b acts as a negative regulator of SIRT1 during adipogenesis [25]. Zerumbone interfered with the binding of miR-146b to the seed sequence of SIRT1 and thus reduced SIRT1 promoterderived luciferase activity (Figure 4C)

A

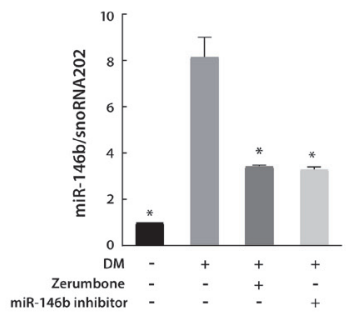

D

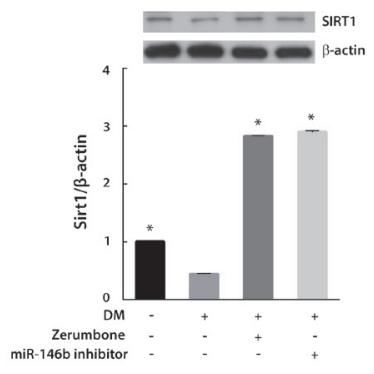

F

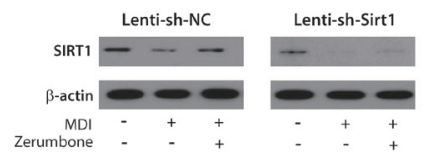

B

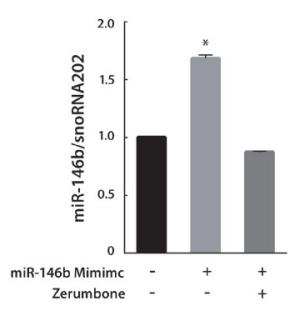

E

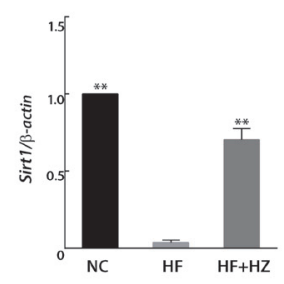

To investigate the mechanisms by which the downregulation of miR-146b by zerumbone affects SIRT1 and the adipogenic response, we measured the SIRT1 mRNA expression and protein levels in the zerumbonetreated or miR-146b inhibitor-treated 3T3-L1 cells. The downregulation of SIRT1 in differentiated 3T3-L1 cells was markedly reversed by treatment with zerumbone and the miR-146b inhibitor $(P<0.05)$ (Figure 4D). The decrease in SIRT1 levels in WAT of HF-fed mice was also
C

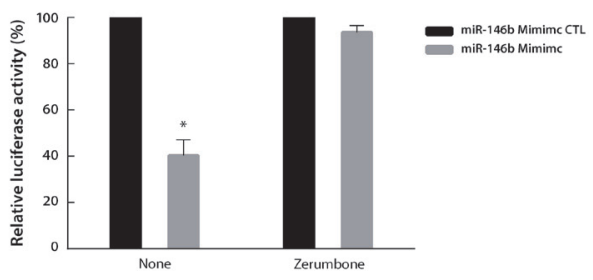

SIRT1 $\beta$-actin

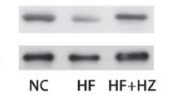

H

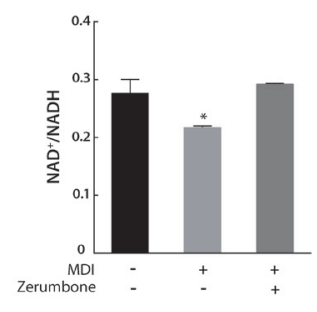

Figure 4: MiR-146b/SIRT1 pathway mediated the anti-adipogenic effect of zerumbone. A. Effects of zerumbone and miR$146 \mathrm{~b}$ inhibitor on miR-146b expression in differentiated 3T3-L1 cells. 3T3-L1 cells were differentiated in the presence of zerumbone (10 $\mu \mathrm{M})$ or miR-146b inhibitor $(10 \mathrm{nM}) .{ }^{*} P<0.05$ versus DM. B. Effect of zerumbone on increased miR-146b expression evoked by treatment with the miR-146b mimic. 3T3-L1 cells were incubated in the presence of or miR-146b activator $(10 \mathrm{nM})$ or zerumbone $(10 \mu \mathrm{M})$ at day 3 for 48 h. $* P<0.05$ versus control. C. Effect of zerumbone on binding of miR-146b to the 3'-UTR of SIRT1 in 3T3-L1 cells. ${ }^{*} P<0.05$ versus miR-146b mimic control with no treatment. D. Effect of zerumbone on SIRT1 mRNA and protein expression in differentiated 3T3L1 cells. $* P<0.05$ versus DM. E. Effect of zerumbone on SIRT1 mRNA and protein expressions in WAT from mice fed NC, HF, and HF + HZ. $* * P<0.01$ versus HF. F. SIRT1 protein expression was measured by western blot. Preadipocytes transduced with Lenti-sh-NC or Lenti-sh-Sirt1 were differentiated in the presence of $10 \mu \mathrm{M}$ zerumbone at day 3 for $48 \mathrm{~h}$. G. Intracellular lipid accumulation was detected by Oil Red O staining and quantified spectrophotometrically in Lenti-sh-NC- or Lenti-sh-Sirt1-transduced 3T3-L1 cells. ${ }^{*} P<0.05$ versus differentiated Lenti-sh-NC, \# $P<0.05$ versus differentiated Lenti-sh-Sirt1. H. Effect of zerumbone on $\mathrm{NAD}^{+} / \mathrm{NADH}$ ratio in differentiated 3T3-L1 cells. $* P<0.05$ versus control. 
attenuated by zerumbone treatment (Figure 4E).

To determine whether the anti-adipogenic effect of zerumbone is mediated by SIRT1, we treated SIRT1knockdown 3T3-L1 cells, generated using a lentiviralbased SIRT1 shRNA (Lenti-sh-Sirt1), with zerumbone (Figure 4F). Zerumbone failed to inhibit adipogenesis in SIRT1-knockdown 3T3-L1 cells (Figure 4G).

SIRT1 is a $\mathrm{NAD}^{+}$-dependent deacetylase that matches the energy output to the energy requirement in the cell by sensing changes in intracellular $\mathrm{NAD}^{+}$ [36]. The NAD biosynthesis pathway is mediated by nicotinamide phosphoribosyltransferase (NAMPT), the rate-limiting enzyme for nicotinamide mononucleotide [37]. Zerumbone was also found to augment SIRT1 activity by increasing the $\mathrm{NAD}^{+} / \mathrm{NADH}$ ratio (Figure $4 \mathrm{H}$ ). Additionally, zerumbone upregulated NAMPT mRNA in 3T3-L1 adipocytes (Figure S1). These results indicate that SIRT1 mediates the anti-adipogenic effect of zerumbone.

\section{Zerumbone increased deacetylation of FOXO1 and PGG1- $\alpha$}

SIRT1 promotes gene transcription by deacetylating specific transcription factors such as forkhead box $\mathrm{O} 1$ (FOXO1) [38] and peroxisome proliferator-activated receptor gamma coactivator 1-alpha (PGC1- $\alpha$ ) [39]. To further investigate the mechanism by which zerumbone regulates the miR-146b/SIRT1 axis to reduce obesity and adipogenesis, we analyzed its effect on the acetylation of FOXO1 and PGC1- $\alpha$, two downstream targets of SIRT1. As shown in Figure 5A, adipogenic stimulation increased the acetylation of FOXO1 and PGC1- $\alpha$. This increase was clearly inhibited following treatment with zerumbone and the miR-146b inhibitor. Similarly, the HF feeding-induced increase in the acetylation of FOXO1 and PGC1- $\alpha$ was markedly reduced by zerumbone in WAT (Figure 5B). These data support the idea that SIRT1 regulates the action of zerumbone via deacetylation of FOXO1 and PGC1- $\alpha$.

A

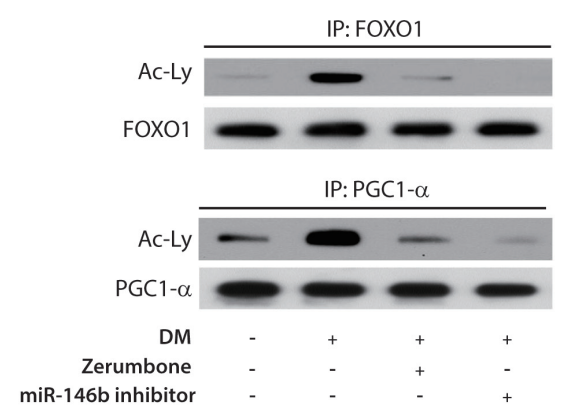

\section{DISCUSSION}

In the present study, we showed that zerumbone protected mice from HF-induced obesity and improved impaired lipid metabolism in WAT via AMPK phosphorylation. Zerumbone also ameliorated dysregulated miRNA profiles in obese WAT tissues and in mature 3T3-L1 cells, with the most prominent change observed in the expression of miR-146b. Zerumbone acts as a miR-146b inhibitor, increasing SIRT1 expression followed by deacetylation of FOXO1 and PGC1- $\alpha$. The proposed mechanism underlying the amelioration of HFevoked metabolic dysfunction by zerumbone in WAT is summarized in Figure 6.

We previously reported that zerumbone mitigates high-fat diet-induced obesogenic effects [25]. However, the exact molecular mechanism by which zerumbone alleviates metabolic disorders has not been reported to date. In this study, we found that zerumbone prevented the development of diet-induced adiposity and inhibited 3T3-L1 differentiation. Zerumbone induced AMPK phosphorylation and decreased adiposity-related upregulation of miR-146b. Thus, zerumbone induced AMPK activation in WAT and improved lipid metabolism through upregulation of fatty oxidation-regulated genes and downregulation of lipogenesis-related genes.

SIRT1 activation results in beneficial effects in metabolic disorders involving obesity, type 2 diabetes, and cardiovascular disease [40]. Therefore, activating SIRT1 pharmacologically should be beneficial in treating obesity and age-related diseases. Regulation of SIRT1 by miRNA has been suggested [41]. Downregulation of miR-34a has been shown to increase the expression of the fibroblast growth factor 21 receptor complex and SIRT1, contributing to the activation of browning in adipose tissue [42]. MiR-34a also reduces $\mathrm{NAD}^{+}$levels and SIRT1 activity by directing targeting NAMPT [43]. In recent reports, miR-3a has been reported to target SIRT1 to inhibit prostate cancer cell proliferation [44].

\section{B}

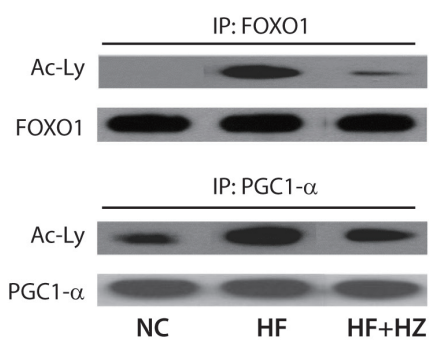

Figure 5: Zerumbone increased deacetylation of FOXO1 and PGC1- $\alpha$. Immunoprecipitation was performed to measure the effect of zerumbone on acetylation of the SIRT1 targets, FOXO1 and PGC1- $\alpha$, in differentiated 3T3- L1 cells A. and WAT from mice B. 
Furthermore, miR-212 negatively regulates autophagy by inhibiting SIRT1 [45]. In addition, we previously reported that miR-146b promotes adipogenesis and obesity by downregulating SIRT1 [25]. In this study, we observed a decrease in miR-146b levels and a subsequent increase in SIRT1 expression in zerumbone-treated 3T3-L1 cells. Furthermore, the anti-adipogenic effect of zerumbone was abolished in 3T3-L1 cells with SIRT1 knockdown, suggesting that zerumbone induced SIRT1 expression by downregulating miR-146b and increasing the $\mathrm{NAD}^{+} /$ $\mathrm{NADH}$ ratio. Upregulation of NAMPT by zerumbone was also observed in adipocytes. Zerumbone supplementation increased SIRT1 activity, ameliorated HF-induced obesity, and reduced HF-induced WAT hypertrophy and hyperplasia in C57BL/6N mice. We additionally observed miR-146b downregulation and subsequent SIRT1 upregulation in the WAT of zerumbone-supplemented mice. Increased SIRT1 expression has additionally been observed in the adipose tissue of obese patients following weight loss [46].

SIRT1 regulates gene transcription by deacetylating specific transcription factors, including FOXO1 [47] and PGC1- $\alpha$ [48]. SIRT1-induced deacetylation of FOXO1 also decreases the expression of adipogenesis-related genes [25]. We found that zerumbone deacetylated FOXO1, which led to downregulation of adipogenesis-related genes in adipose tissue. SIRT1 catalyzes deacetylation of PGC1 $\alpha$, leading to induction of uncoupling protein 1 (UCP1), which is involved in thermogenesis regulation [49].

The miR-146b has been reported to serve as a prognostic marker for non-small lung cancer [50]. The miR-146b inhibits metastasis in glioma [51] and breast cancer cells [52], rescues hypoxia-induced apoptosis in cardiomyocytes [53], and ameliorates retinal inflammation in diabetes [54]. Previously, we demonstrated that inhibition of miR-146b alleviates diet-induced obesity through SIRT1 regulation. In this study, we attempted to identify a new compound involved in the miR-146b/ SIRT1 pathway, and found that zerumbone is a candidate for prevention of obesity.

In summary, we showed that zerumbone exerts protective effects against HF-induced obesity and inhibits adipocyte differentiation. Moreover, zerumbone improves impaired lipid metabolism by increasing fatty acid oxidation and reducing lipogenesis via AMPK phosphorylation. The downregulation of miR-146b observed following zerumbone treatment resulted in increased mRNA and protein expression of SIRT1. The consequent deacetylation of $\mathrm{FOXO} 1$ and $\mathrm{PGC} 1-\alpha$ resulted in reduced adipogenesis and inhibited weight gain. These results indicate that zerumbone may serve as a potential therapeutic agent by inhibiting adipogenesis and protecting against high-fat diet induced obesity.

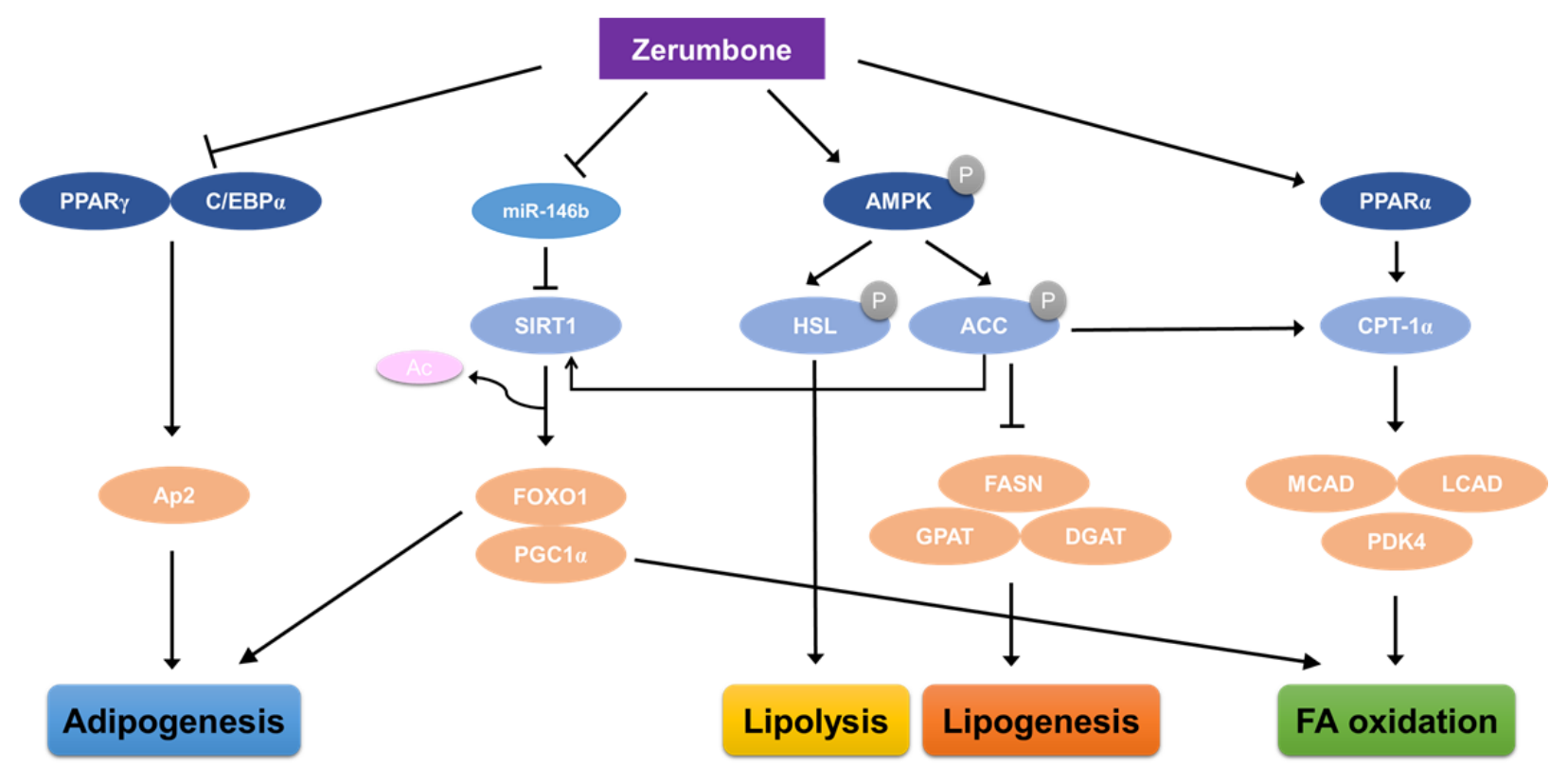

Figure 6: Proposed mechanism through which zerumbone improves diet-induced adiposity in WAT via the AMPK and miR-146b/SIRT1 pathways. Zerumbone induces phosphorylation of AMPK and, following phosphorylation of ACC, induces increased fatty acid oxidation and reduced lipogenesis. Zerumbone also acts as a miR-146b inhibitor and downregulates miR-146b, leading to SIRT1 activation. This results in the deacetylation of the SIRT1 targets, FOXO1 and PGC1- $\alpha$, and improves adiposity by increasing fat mobilization and improving lipid metabolism. 


\section{MATERIALS AND METHODS}

\section{Animals}

Five-week-old male C57BL/6N mice were obtained from Orient Bio Inc. (Seongnam, Korea). Animals were housed at a temperature of $24 \pm 2{ }^{\circ} \mathrm{C}$ in $50-60 \%$ relative humidity, with $12 \mathrm{~h} \mathrm{light/dark} \mathrm{cycles} \mathrm{throughout} \mathrm{the}$ experiment. Water and food were supplied ad libitum. After 1 week of acclimation, the mice were randomly divided into four groups ( $n=10$ per group): (1) NC (10\% of total calories from fat), (2) HF ( $45 \%$ of total calories from fat), (3) HF containing $0.01 \%$ zerumbone (HF + LZ), and (4) HF containing $0.025 \%$ zerumbone ( $\mathrm{HF}+\mathrm{HZ}$ ). The composition of the experimental diets is shown in Table S1. All diets were based on the AIN-76A diet [55]. Body weights were recorded weekly. After 8 weeks on the diet, the mice were euthanized by cervical dislocation. All animal experiments in this study were conducted in accordance with a protocol approved by the Korea Food Research Institute-Institutional Animal Care and Use Committee (KFRI-IACUC No. 2013-149).

\section{Histological examination}

Tissues were fixed in 10\% buffered formalin, embedded in paraffin, sectioned, and stained with hematoxylin and eosin (H\&E) for histological analyses. The stained areas were observed using a light microscope (Olympus, Tokyo, Japan) with a magnification of $200 \times$. The mean adipocyte size was quantified using ImageJ software (NIH, Bethesda, MD, USA).

\section{Serum and liver biochemistry}

Serum glucose, triglyceride (TG), total cholesterol (TC), high-density lipoprotein (HDL), and free fatty acid (FFA) levels were measured enzymatically as previously described [56]. Serum insulin and leptin levels were measured using an ELISA kit (ALPCO Diagnostics, Salem, NH). To measure hepatotoxicity, the AST and ALT activity of liver homogenates was measured.

\section{Quantitative real-time polymerase chain reaction}

Total RNA was isolated using a NucleoSpin RNA II kit (Macherey-Nagel, Duren, Germany). For miRNA measurement, total RNA was reverse transcribed using the TaqMan MicroRNA reverse transcription kit and analyzed by real-time PCR using the TaqMan MicroRNA assay kit (Applied Biosystems, Foster City, CA, USA). Expression of miRNA was normalized to that of endogenous snoRNA202. For the mRNA expression assay, qRT-
PCR was performed using SYB Green PCR Master Mix in a StepOnePlus Real-Time PCR system (Applied Biosystems). The level of each mRNA was normalized to that of $\beta$-actin. The oligonucleotide sequences for each primer are listed in S2 Table.

\section{Western blot}

Cells and WAT were lysed with RIPA buffer. Western blot analysis was performed as previously described [57]. Blots were probed with primary antibodies against PGC1- $\alpha, \mathrm{C} / \mathrm{EBP} \alpha$, FASN, Ap2 (Cell Signaling, Danvers, MA), PPAR $\gamma$, SIRT1, FOXO1, $\beta$-actin (Santa Cruz Biotechnology Inc., Santa Cruz, CA).

\section{Cell culture and differentiation}

3T3-L1 fibroblasts (ATCC, Manassas, VA) or transduced cells were maintained and differentiated as described previously [57]. Briefly, 3T3-L1 cells were maintained in Dulbecco's modified Eagle medium (DMEM) containing $25 \mathrm{mM}$ glucose, $10 \%$ calf serum, $100 \mathrm{U} / \mathrm{mL}$ penicillin, $100 \mu \mathrm{g} / \mathrm{mL}$ streptomycin, and $4 \mathrm{mM}$ L-glutamine. The cytotoxic effects of zerumbone (>98\%, Z3902, Sigma Aldrich, St. Louis, MO) against 3T3-L1 cells were determined using the 3-[4,5-dimethylthiazol2-yl]-2,5 diphenyl tetrazolium bromide (MTT) assay as previously described [58]. Following differentiation, cells were stained with Oil Red O (Sigma-Aldrich), dissolved in isopropanol, and quantified by measuring the optical absorbance at $500 \mathrm{~nm}$. Images were collected using an Olympus microscope at a magnification of $\times 100$ (Tokyo, Japan). To induce SIRT1 knockdown, 3T3-L1 cells were transduced with a lentiviral-based SIRT1 short hairpin RNA (shRNA; Genepharma, Shanghai, China). SIRT1 knockdown was confirmed by immunoblotting with an anti-SIRT1 antibody.

\section{Functional study of miRNA}

To modulate miR-146b expression, we transfected miR-146b-specific inhibitor or activator into 3T3-L1 cells, as previously described [25]. Oligonucleotides $(10 \mathrm{nM})$ were transfected into cells with Lipofectamine RNAiMAX (Invitrogen, Carlsbad, CA). Overexpression or inhibition of miR-146b expression was verified by qRT-PCR. The effect of zerumbone on binding efficacy between miR$146 \mathrm{~b}$ and its target was measured using $3^{\prime}$-UTR luciferase reporter assays, as previously reported by Ahn et al. [25]. Luciferase activity was normalized to the corresponding $\beta$-galactosidase activity. 


\section{$\mathrm{NAD}^{+} / \mathrm{NADH}$ ratio measurement}

The $\mathrm{NAD}^{+} / \mathrm{NADH}$ ratio was measured in wholecell extracts of 3T3-L1 cells using a NAD $/ \mathrm{NADH}$ quantification kit (Biovision, San Francisco, CA, USA). The absorbance was measured at $450 \mathrm{~nm}$ and normalized to protein the concentration.

\section{Immunoprecipitation}

Immunoprecipitation was performed using the protein A immunoprecipitation kit (Roche Applied Science, Mannheim, Germany) by mixing cell lysates with an antibody against FOXO1 and PGC1- $\alpha$. Immunoprecipitated proteins were separated by gel electrophoresis and subjected to western blotting with an antibody against acetylated lysine (Santa Cruz Biotechnology).

\section{Statistical analysis}

Results were expressed as the mean \pm standard deviation (SD). Statistical analyses were performed using GraphPad Prism 6 software (San Diego, CA). One-way analysis of variance (ANOVA) was used to compare quantitative data among groups. The Bonferroni post-hoc test was used if ANOVA indicated significance $(P<0.05)$.

\section{Abbreviations}

$\mathrm{C} / \mathrm{EBP} \alpha$, CCAAT enhancer binding protein alpha; FOXO1, forkhead box O 1; HF, high-fat diet; miRNA, microRNA; PGC-1 $\alpha$, peroxisome proliferator-activated receptor gamma coactivator 1 alpha; SIRT1, sirtuin 1; WAT, white adipose tissue.

\section{Author contributions}

JA and TYH designed the experiments and wrote the paper. JA and HL performed the cell and animal studies. $\mathrm{CHJ}$ conducted western blots. JA and WHC analyzed the data.

\section{CONFLICTS OF INTEREST}

The authors declare no conflict of interest.

\section{FUNDING} Institute.

\section{REFERENCES}

1. Hofbauer KG. Molecular pathways to obesity. Int J Obes Relat Metab Disord. 2002; 26:S18-27.

2. Hubert HB, Feinleib M, McNamara PM, Castelli WP. Obesity as an independent risk factor for cardiovascular disease: a 26-year follow-up of participants in the Framingham Heart Study. Circulation. 1983; 67:968-977.

3. Gloy VL, Briel M, Bhatt DL, Kashyap SR, Schauer PR, Mingrone G, Bucher HC, Nordmann AJ. Bariatric surgery versus non-surgical treatment for obesity: a systematic review and meta-analysis of randomised controlled trials. BMJ. 2013; 347:f5934.

4. Rupasinghe HP, Sekhon-Loodu S, Mantso T, Panayiotidis MI. Phytochemicals in regulating fatty acid beta-oxidation: Potential underlying mechanisms and their involvement in obesity and weight loss. Pharmacol Ther. 2016; 165:153163.

5. Tanaka T, Shimizu M, Kohno H, Yoshitani S, Tsukio Y, Murakami A, Safitri R, Takahashi D, Yamamoto K, Koshimizu K, Ohigashi H, Mori H. Chemoprevention of azoxymethane-induced rat aberrant crypt foci by dietary zerumbone isolated from Zingiber zerumbet. Life Sci. 2001; 69:1935-1945.

6. Prasannan R, Kalesh KA, Shanmugam MK, Nachiyappan A, Ramachandran L, Nguyen AH, Kumar AP, Lakshmanan M, Ahn KS, Sethi G. Key cell signaling pathways modulated by zerumbone: role in the prevention and treatment of cancer. Biochem Pharmacol. 2012; 84:12681276.

7. Jegannathan SD, Arul S, Dayalan H. Zerumbone, a Sesquiterpene, Controls Proliferation and Induces Cell Cycle Arrest in Human Laryngeal Carcinoma Cell Line Hep-2. Nutr Cancer. 2016; 68:865-872.

8. Kang CG, Lee HJ, Kim SH, Lee EO. Zerumbone Suppresses Osteopontin-Induced Cell Invasion Through Inhibiting the FAK/AKT/ROCK Pathway in Human Non-Small Cell Lung Cancer A549 Cells. J Nat Prod. 2016; 79:156-160.

9. Wang C, Zou S, Cui Z, Guo P, Meng Q, Shi X, Gao Y, Yang G, Han Z. Zerumbone protects INS-1 rat pancreatic beta cells from high glucose-induced apoptosis through generation of reactive oxygen species. Biochem Biophys Res Commun. 2015; 460:205-209.

10. Shamoto T, Matsuo Y, Shibata T, Tsuboi K, Nagasaki T, Takahashi H, Funahashi H, Okada Y, Takeyama H. Zerumbone inhibits angiogenesis by blocking NF-kappaB activity in pancreatic cancer. Pancreas. 2014; 43:396-404.

11. Chen BY, Lin DP, Su KC, Chen YL, Wu CY, Teng MC, Tsai YT, Sun CY, Wang SR, Chang HH. Dietary zerumbone prevents against ultraviolet B-induced cataractogenesis in the mouse. Mol Vis. 2011; 17:723-730.

12. Ozaki Y, Kawahara N, Harada M. Anti-inflammatory effect of Zingiber cassumunar Roxb. and its active principles. Chem Pharm Bull (Tokyo). 1991; 39:2353-2356. 
13. Murakami A, Takahashi M, Jiwajinda S, Koshimizu $\mathrm{K}$, Ohigashi H. Identification of zerumbone in Zingiber zerumbet Smith as a potent inhibitor of 12-O-tetradecanoylphorbol-13-acetate-induced EpsteinBarr virus activation. Biosci Biotechnol Biochem. 1999; 63:1811-1812.

14. Tzeng TF, Liou SS, Chang CJ, Liu IM. Zerumbone, a Natural Cyclic Sesquiterpene of Zingiber zerumbet Smith, Attenuates Nonalcoholic Fatty Liver Disease in Hamsters Fed on High-Fat Diet. Evid Based Complement Alternat Med. 2013; 2013:303061.

15. Beattie JH, Nicol F, Gordon MJ, Reid MD, Cantlay L, Horgan GW, Kwun IS, Ahn JY, Ha TY. Ginger phytochemicals mitigate the obesogenic effects of a high-fat diet in mice: a proteomic and biomarker network analysis. Mol Nutr Food Res. 2011; 55:S203-213.

16. Tzeng TF, Liou SS, Chang CJ, Liu IM. Zerumbone, a tropical ginger sesquiterpene, ameliorates streptozotocininduced diabetic nephropathy in rats by reducing the hyperglycemia-induced inflammatory response. Nutr Metab (Lond). 2013; 10:64.

17. Bartel DP. MicroRNAs: genomics, biogenesis, mechanism, and function. Cell. 2004; 116:281-297.

18. Acunzo M, Romano G, Wernicke D, Croce CM. MicroRNA and cancer-a brief overview. Adv Biol Regul. 2015; 57:19 .

19. Wienholds E, Plasterk RH. MicroRNA function in animal development. FEBS letters. 2005; 579:5911-5922.

20. Xu P, Vernooy SY, Guo M, Hay BA. The Drosophila microRNA Mir-14 suppresses cell death and is required for normal fat metabolism. Current biology : CB. 2003; 13:790795.

21. Takanabe R, Ono K, Abe Y, Takaya T, Horie T, Wada H, Kita T, Satoh N, Shimatsu A, Hasegawa K. Up-regulated expression of microRNA-143 in association with obesity in adipose tissue of mice fed high-fat diet. Biochemical and biophysical research communications. 2008; 376:728-732.

22. Krutzfeldt J, Stoffel M. MicroRNAs: a new class of regulatory genes affecting metabolism. Cell Metab. 2006; 4:9-12.

23. Kim SY, Kim AY, Lee HW, Son YH, Lee GY, Lee JW, Lee YS, Kim JB. miR-27a is a negative regulator of adipocyte differentiation via suppressing PPARgamma expression. Biochem Biophys Res Commun. 2010; 392:323-328.

24. Zou B, Ge Z, Zhu W, Xu Z, Li C. Persimmon tannin represses 3T3-L1 preadipocyte differentiation via upregulating expression of miR-27 and down-regulating expression of peroxisome proliferator-activated receptorgamma in the early phase of adipogenesis. Eur J Nutr. 2015; 54:1333-1343.

25. Ahn J, Lee H, Jung CH, Jeon TI, Ha TY. MicroRNA-146b promotes adipogenesis by suppressing the SIRT1-FOXO1 cascade. EMBO Mol Med. 2013; 5:1602-1612.

26. Ozer J, Ratner M, Shaw M, Bailey W, Schomaker S.
The current state of serum biomarkers of hepatotoxicity. Toxicology. 2008; 245:194-205.

27. Sullivan JE, Brocklehurst KJ, Marley AE, Carey F, Carling $\mathrm{D}$, Beri RK. Inhibition of lipolysis and lipogenesis in isolated rat adipocytes with AICAR, a cell-permeable activator of AMP-activated protein kinase. FEBS Lett. 1994; 353:33-36.

28. Saha AK, Schwarsin AJ, Roduit R, Masse F, Kaushik V, Tornheim K, Prentki M, Ruderman NB. Activation of malonyl-CoA decarboxylase in rat skeletal muscle by contraction and the AMP-activated protein kinase activator 5-aminoimidazole-4-carboxamide-1-beta -D-ribofuranoside. The Journal of biological chemistry. 2000; 275:24279-24283.

29. Sakinah SA, Handayani ST, Hawariah LP. Zerumbone induced apoptosis in liver cancer cells via modulation of Bax/Bcl-2 ratio. Cancer cell international. 2007; 7:4.

30. Xian $M$, Ito $K$, Nakazato $T$, Shimizu $T$, Chen $C K$, Yamato K, Murakami A, Ohigashi H, Ikeda Y, Kizaki M. Zerumbone, a bioactive sesquiterpene, induces G2/M cell cycle arrest and apoptosis in leukemia cells via a Fas- and mitochondria-mediated pathway. Cancer science. 2007; 98:118-126.

31. McGregor RA, Choi MS. microRNAs in the regulation of adipogenesis and obesity. Curr Mol Med. 2011; 11:304316.

32. Arner P, Kulyte A. MicroRNA regulatory networks in human adipose tissue and obesity. Nat Rev Endocrinol. 2015; 11:276-288.

33. Hilton C, Neville MJ, Karpe F. MicroRNAs in adipose tissue: their role in adipogenesis and obesity. Int J Obes. $2013 ; 37: 325-332$.

34. Chartoumpekis DV, Zaravinos A, Ziros PG, Iskrenova RP, Psyrogiannis AI, Kyriazopoulou VE, Habeos IG. Differential expression of microRNAs in adipose tissue after long-term high-fat diet-induced obesity in mice. PLoS One. 2012; 7:e34872.

35. Bartel DP. MicroRNAs: target recognition and regulatory functions. Cell. 2009; 136:215-233.

36. Michan S, Sinclair D. Sirtuins in mammals: insights into their biological function. The Biochemical journal. 2007; 404:1-13.

37. Revollo JR, Grimm AA, Imai S. The NAD biosynthesis pathway mediated by nicotinamide phosphoribosyltransferase regulates Sir2 activity in mammalian cells. J Biol Chem. 2004; 279:50754-50763.

38. Armoni M, Harel C, Karni S, Chen H, Bar-Yoseph F, Ver MR, Quon MJ, Karnieli E. FOXO1 represses peroxisome proliferator-activated receptor-gamma1 and -gamma2 gene promoters in primary adipocytes. A novel paradigm to increase insulin sensitivity. The Journal of biological chemistry. 2006; 281:19881-19891.

39. Rodgers JT, Lerin C, Gerhart-Hines Z, Puigserver P. Metabolic adaptations through the PGC-1 alpha and SIRT1 
pathways. FEBS letters. 2008; 582:46-53.

40. Imai S, Kiess W. Therapeutic potential of SIRT1 and NAMPT-mediated NAD biosynthesis in type 2 diabetes. Front Biosci (Landmark Ed). 2009; 14:2983-2995.

41. Choi SE, Kemper JK. Regulation of SIRT1 by microRNAs. Mol Cells. 2013; 36:385-392.

42. Fu T, Seok S, Choi S, Huang Z, Suino-Powell K, Xu HE, Kemper B, Kemper JK. MicroRNA 34a inhibits beige and brown fat formation in obesity in part by suppressing adipocyte fibroblast growth factor 21 signaling and SIRT1 function. Molecular and cellular biology. 2014; 34:41304142 .

43. Choi SE, Fu T, Seok S, Kim DH, Yu E, Lee KW, Kang Y, Li X, Kemper B, Kemper JK. Elevated microRNA-34a in obesity reduces NAD+ levels and SIRT1 activity by directly targeting NAMPT. Aging Cell. 2013; 12:1062-1072.

44. Duan K, Ge YC, Zhang XP, Wu SY, Feng JS, Chen SL, Zhang LI, Yuan ZH, Fu CH. miR-34a inhibits cell proliferation in prostate cancer by downregulation of SIRT1 expression. Oncol Lett. 2015; 10:3223-3227.

45. Ramalinga M, Roy A, Srivastava A, Bhattarai A, Harish V, Suy S, Collins S, Kumar D. MicroRNA-212 negatively regulates starvation induced autophagy in prostate cancer cells by inhibiting SIRT1 and is a modulator of angiogenesis and cellular senescence. Oncotarget. 2015; 6:34446-34457. doi: 10.18632/oncotarget.5920.

46. Moschen AR, Wieser V, Gerner RR, Bichler A, Enrich B, Moser P, Ebenbichler CF, Kaser S, Tilg H. Adipose tissue and liver expression of SIRT1, 3, and 6 increase after extensive weight loss in morbid obesity. Journal of hepatology. 2013; 59:1315-1322.

47. Daitoku H, Hatta M, Matsuzaki H, Aratani S, Ohshima T, Miyagishi M, Nakajima T, Fukamizu A. Silent information regulator 2 potentiates Foxo1-mediated transcription through its deacetylase activity. Proceedings of the National Academy of Sciences of the United States of America. 2004; 101:10042-10047.

48. Nemoto S, Fergusson MM, Finkel T. SIRT1 functionally interacts with the metabolic regulator and transcriptional coactivator PGC-1\{alpha\}. The Journal of biological chemistry. 2005; 280:16456-16460.
49. Bostrom P, Wu J, Jedrychowski MP, Korde A, Ye L, Lo JC, Rasbach KA, Bostrom EA, Choi JH, Long JZ, Kajimura S, Zingaretti MC, Vind BF, et al. A PGC1-alpha-dependent myokine that drives brown-fat-like development of white fat and thermogenesis. Nature. 2012; 481:463-468.

50. Raponi M, Dossey L, Jatkoe T, Wu X, Chen G, Fan H, Beer DG. MicroRNA classifiers for predicting prognosis of squamous cell lung cancer. Cancer Res. 2009; 69:57765783.

51. Xia H, Qi Y, Ng SS, Chen X, Li D, Chen S, Ge R, Jiang S, Li G, Chen Y, He ML, Kung HF, Lai L, Lin MC. microRNA-146b inhibits glioma cell migration and invasion by targeting MMPs. Brain Res. 2009; 1269:158165.

52. Bhaumik D, Scott GK, Schokrpur S, Patil CK, Campisi J, Benz CC. Expression of microRNA-146 suppresses NFkappaB activity with reduction of metastatic potential in breast cancer cells. Oncogene. 2008; 27:5643-5647.

53. Li JW, He SY, Feng ZZ, Zhao L, Jia WK, Liu P, Zhu Y, Jian Z, Xiao YB. MicroRNA-146b inhibition augments hypoxia-induced cardiomyocyte apoptosis. Mol Med Rep. 2015; 12:6903-6910.

54. Fulzele S, El-Sherbini A, Ahmad S, Sangani R, Matragoon S, El-Remessy A, Radhakrishnan R, Liou GI. MicroRNA$146 \mathrm{~b}-3 \mathrm{p}$ regulates retinal inflammation by suppressing adenosine deaminase-2 in diabetes. Biomed Res Int. 2015; 2015:846501.

55. Bieri JG. AIN-76 diet. J Nutr. 1979; 109:925-926.

56. Um MY, Ahn J, Jung CH, Ha TY. Cholesterol-lowering Effect of Rice Protein by Enhancing Fecal Excretion of Lipids in Rats. Preventive nutrition and food science. 2013; 18:210-213.

57. Ahn J, Lee H, Kim S, Ha T. Curcumin-induced suppression of adipogenic differentiation is accompanied by activation of Wnt/beta-catenin signaling. Am J Physiol Cell Physiol. 2010; 298:C1510-1516.

58. Park MJ, Han JS. Protective Effects of the Fermented Laminaria japonica Extract on Oxidative Damage in LLCPK1 Cells. Preventive nutrition and food science. 2013; 18:227-233. 